Conclusion Intra-abdominal tumors are more common in males Neuroblastoma was the most common tumor. Most of the tumors were noted in children less than 5 years of age.

\section{CLINICOPATHOLOGICAL ASPECTS OF LOWER GASTROINTESTINAL BLEEDING IN CHILDREN: A SINGLE CENTER EXPERIENCE FROM SOUTHERN IRAN}

doi:10.1136/archdischild-2012-302724.0681

E Fallahzadeh, M Zahmatkeshan, KS Najib, B Geramizadeh, M Haghighat, MHI Imanieh, SM Dehghani. Shiraz University of Medical Sciences, Shiraz, Iran

Purpose To determine the common etiologies and characteristics of lower gastrointestinal bleeding in children in southern Iran.

Methods This study was performed from March 2006 to March 2011 in Nemazee Hospital. All pediatric patients who referred to our center with visible lower gastrointestinal bleeding or two consecutive positive occult blood tests with at least one week interval were included in the study. The patients were categorized as neonates (1-28 days), infants (29 days-2 years), children (2-10 years) and adolescents ( $>10$ years) and the findings were reported separately in each group. All the patients underwent colonoscopy and several mucosal biopsies where taken. Demographic and clinical information as well as colonoscopy and pathology findings were reported.

Results We included 363 pediatric patients with a mean age of $71.9 \pm 58.4$ months, ranging from 1 to 216 months. There were 215 $(59.2 \%)$ boys and 148 (40.8\%) girls. The most common colonoscopy finding was sigmoid colon polyp in $91(25.1 \%)$ patients followed by descending colon petechia in $78(21.5 \%)$ patients, rectal whitish lesions in $45(12.4 \%)$ patients, and sigmoid and rectal ulcers in 37 $(10.2 \%)$ patients. Biopsy samples were non-specific in $96(26.4 \%)$ patients. The most common pathological finding was juvenile polyp in $84(23.1 \%)$ patients followed by lymphoid nodular hyperplasia in $55(15.2 \%)$ patients and solitary rectal ulcers in $25(6.9 \%)$ patients. Conclusions Lower GI bleeding is more common among 2-10 year-old children and is rarely encountered in neonates. Hematochezia was the most common form of presentation followed by bloody diarrhea and occult blood.

\section{THE ROLE OF LACTOBACILLUS RHAMNOSUS GG SUPPLEMENTATION ON THE ERADICATION OF PATHOGENIC INTESTINAL FLORA IN INFANTS}

doi:10.1136/archdischild-2012-302724.0682

W Furmaga-Jablonska, K Czyz, M Rymgayllo-Katska, G Polkowska, M Zatorska-Karpus. Department of Neonate and Infant Pathology, Medical University, Lublin, Poland

Objectives and study It is suggested that colonization of gastrointestinal tract by pathological microorganisms in infants may modulate local mucosal immune response and start inflammation. Disorders of the digestive system in infants may be manifested by diarrhea, blood in the stools or abdominal pain. Probiotics, which exert effect on the health and well-being of the host, may be in that situations, a potential therapeutic option. Therefore, we constructed the study to investigate the efficacy of Lactobacillus rhamnosus GG (LGG) supplementation for the eradication of pathogenic intestinal flora in infants.

Methods A randomized, double-blind, placebo controlled trial included 65 infants (31 girls and 34 boys) at age from 2-st to 20-th month. All children presented symptoms such as vomiting, diarrhea, dyspeptic stools or blood in the stools. The enteropathological bacterial flora were revealed in each cases. Infants were randomly allocated to receive Lactobacillus GG $3 \times 10^{9} \mathrm{CFU}$ (active LGG group, $\mathrm{n}=36$ ) or placebo (maltodextrin) (placebo group, $\mathrm{n}=29$ ). After 14 and 28 days of oral supplementation control stool cultures were performed.
Results Compared to the placebo group, infants in LGG group had an increase in benefit of the pathological intestinal flora eradication after 14 days and 28 days of oral LGG supplementation (RR 1.31, NNT 12, RR 1.39 NNT 4) respectively.

Conclusion The use of LGG supplementation seems to be effective in the eradication of gastrointestinal colonization by pathological bacteria.

\section{IS ESOPHAGEAL GLYCOGENIC ACANTHOSIS ASSOCIATED WITH GASTROESOPHAGEAL REFLUX DISEASE IN CHILDREN?}

doi:10.1136/archdischild-2012-302724.0683

${ }^{1} \mathrm{HH}$ Emiroglu, 'S Sokucu, 'S Cantez, ${ }^{2} \mathrm{M}$ Gulluoglu, ${ }^{3} \mathrm{M}$ Keser, ${ }^{1} 0$ Durmaz. ${ }^{1}$ Pediatric Gastroenterology, Hepatology and Nutrition; ${ }^{2}$ Pediatric Pathology; ${ }^{3 P e d i a t r i c ~ I n f e c t i o u s ~}$ Diseases, Istanbul University Istanbul School of Medicine, Istanbul, Turkey

Background and Aim Glycogenic acanthosis $(G A)$ is a common benign endoscopic and histopathological finding which has not known the pathogenesis and aetiology. There is a study about the relationship between $G A$ and gastroesophageal reflux disease (GERD) on adults in the literature.

Retrospective in nature the aim of our study was to find out whether GA is associated with GERD in children.

Methods A total of 213 children (101 females, average age 8.4 \pm 4.9 years, range 2 months-18 years), who underwent diagnostic esophagogastroduodenoscopy during a three years period were included in this study.

The histopathological findings of GA and reflux-related changes according to Knuff \& Leape classification were examined by the same pathologist. SPSS for Windows Release 16.0 was used to analyse the statistical data. All tests of statistical significance were twosided with a p-value $<0.05$.

Results Of the 213 children, 67 (31.4\%) had GERD. GA was found in $38(17.8 \%)$ of the 213 children. The prevalence of GA in the patients with GERD (12 of 67, 17.9\%) and that in without (26 of $146,17.8 \%$ ) were similar. Likewise, when the prevalence of GERD in GA-positive patients (12 of $38,31.6 \%$ ) was compared with that in GA-negative patients (55 of $175,31.4 \%$ ), no statistically significant difference was found. It was found out that the prevalence of nonerosive reflux disease was quite alike in GA-positive and GA-negative children.

Conclusion Presence of GA was not associated with GERD in children.

\section{GLYCOGENIC ACANTHOSIS OF THE ESOPHAGUS: IS IT ASSOCIATED WITH HELICOBACTER PYLORI INFECTION IN CHILDREN?}

doi:10.1136/archdischild-2012-302724.0684

${ }^{1} \mathrm{HH}$ Emiroglu, 'S Sokucu, ${ }^{2} \mathrm{M}$ Gulluoglu, ${ }^{1} \mathrm{~S}$ Cantez, ${ }^{3} \mathrm{M}$ Keser, ${ }^{10}$ Durmaz. ${ }^{1}$ Pediatric Gastroenterology, Hepatology and Nutrition; ${ }^{2}$ Pediatric Pathology; ${ }^{3}$ Pediatric Infectious Diseases, Istanbul University Istanbul School of Medicine, Istanbul, Turkey

Background and Aim Glycogenic acanthosis (GA) is usually reported as a histologic finding. The pathogenesis and aetiology of $G A$ has not been explained definitively yet.

There are many studies about the relationship between Helicobacter pylori (H. pylori) infection and gastric or extragastric manifestations of malignant or non-malignant diseases in the literature. However, only one study deals with the association between GA and $H$. pylori infection.

The aim of our study was to find out whether $G A$ is associated with $H$. pylori infection in children.

Methods A total of 206 children ( 98 female, median age 9.0 years, range 2 months-18 years), who underwent diagnostic esophagogastroduodenoscopy during a three years period, were included. GA 
diagnosis was based on histopathological findings seen in the distal oesophageal mucosa. The diagnosis of $H$. pylori infection was made if rapid urease test and histological examination of gastric biopsies obtained during endoscopy were both positive. All the findings were retrospectively examined. The Statistical Package for the Social Sciences for Windows Release 16.0 was used to analyse the statistical data.

Results Of the 206 children, $70(34.0 \%)$ had GA. H. pylori infection was found in $72(35 \%)$ children. No significant difference was found when the prevalence of $H$. pylori infection in patients with GA (24 of $70,34.3 \%$ ) was compared with that in patients without GA (48 of $136,35.3 \%)$.

Conclusion No evidence has been found in this pediatric study to support the view that there might be an association between $G A$ and $H$. pylori infection.

\section{FOOD ALLERGY - GASTRO-ESOPHAGEAL REFLUX DISEASE ASSOCIATION IN INFANTS}

doi:10.1136/archdischild-2012-302724.0685

C Cazan, L Dobrota, ML Neamtu. Pediatric Clinic Hospital, Lucian Blaga University of Sibiu, Sibiu, Romania

Background Cow's milk allergy is considered to be the first and most common type of allergy during early infancy. Gastroesophageal reflux disease and cow's milk allergy are two different diseases with common clinical features.

The aim of the study was to evaluate the clinical data in relation with gastroesophageal reflux and cow's milk allergy in infants.

Material and Methods The prospective study includes 36 infants aged between 2-6 months who attended at Pediatric Clinic during the year 2011 for the clinical evocative manifestations of gastroesophageal reflux. Study protocol includes: clinical criteria, familial/ personal atopic features, mother diet, duration of breastfeeding, infant formula, esophageal ultrasonographic study, serum specific IgE cow's milk.

Results The clinical presentations of the infants were associated or isolated agitation/irritability, feeding refusal $(p<0.0002)$, poor weight gain $(p<0.0001)$, vomiting, wheezing, apnea and atopic dermatitis. Specific Ig E revealed allergy in 10 cases. The implication of cow's milk allergy was in 10/36 cases (27.7\%). The key elements evocating the link between cow's milk allergy and gastroesophageal reflux were the persistence of symptoms under anti-reflux therapy and the improvement of symptoms under the exclusion of cow's milk. Favorable clinical course, disappearance of symptoms, weight gain under anti-reflux therapy confirmed the gastroesophagial reflux in the other cases. Esophageal ultrasonography was a useful noninvasive test in patients with reflux.

Conclusions Clinical assessment and allergy tests in infants with the suspicion of gastroesophageal reflux revealed the association of these diseases. The concomitant therapy was followed by clinical resolution of symptoms.

\section{NUCLEAR TRANSIT SCINTIGRAPHY (NTS) - AN EVOLVING ROLE FROM DIAGNOSTIC TO MONITORING TOOL IN CHILDREN WITH CHRONIC CONSTIPATION}

doi:10.1136/archdischild-2012-302724.0686

${ }^{1,2,3} \mathrm{Y}$ Y Yik, ${ }^{4} \mathrm{DJ}$ Cook, ${ }^{4} \mathrm{DM}$ Veysey, ${ }^{4} \mathrm{CF}$ Tudball, ${ }^{4} \mathrm{BS}$ King, ${ }^{4} \mathrm{KA}$ Morris, ${ }^{4} \mathrm{TM}$ Cain, ${ }^{3}$ BR Southwell, $2,3,5 \mathrm{JM}$ Hutson. 'Department of General Surgery, Faculty of Medicine, University of Malaya, Kuala Lumpur, Malaysia; '2Department of Paediatrics, University of Melbourne; ${ }^{3}$ Gut Motility and Research Laboratory, Murdoch Children's Research Institute; ${ }^{4}$ Department of Biomedical Imaging; ${ }^{5}$ Department of Urology, Royal Childrens Hospital Melbourne, Melbourne, VIC, Australia

Purpose Nuclear transit scintigraphy (NTS) is a relatively new investigation for children with intractable chronic constipation.
Three patterns of colonic transit were identified: normal, slow and rapid colonic transit. We aimed to determine the use of NTS over the last 12 years at a tertiary institute. We hypothesised that NTS has evolved from a diagnostic to a monitoring tool.

Methods NTS were reviewed retrospectively (1999-2011) and characterized based on 3 different colonic transit patterns with further division into a new/repeat study (Ethics30059A). Statistical analysis was performed with Chi-square test to examine the effect of change; $p<0.05$ considered significant. Transcutaneous electrical stimulation (TES) was introduced to treat slow-transit constipation (STC) since 2006. Hence, we examined the changing role of NTS before and after TES use in STC children.

Results From 1999-2011, there were 955 NTS performed (667 new and 288 repeat studies): normal colonic transit - 133 new and 27 repeat; rapid colonic transit - 190 new and 24 repeat; slow colonic transit - 344 new and 237 repeat studies; with more repeat studies for STC children ( $p<0.0001$, Chi-square). There was an increase of repeat studies from 1999-2005 (15\%) to 2006-2011 (30\%, p<0.0001). Since 2006, 95/237 (40\%) NTS performed were repeat studies to monitor the effects of TES in STC children.

Conclusion NTS has is a useful diagnostic tool and helps to improve management of chronic constipation by guiding therapy, targeting the underlying dysmotility. It also provides objective assessment in monitoring response to therapy/intervention.

\section{ROLE OF INTRAUTERINE CMV INFECTION IN FORMATION OF BILIARY ATRESIA}

doi:10.1136/archdischild-2012-302724.0687

${ }^{1}$ AV Degtyareva, ${ }^{2} E L$ Tumanova, ${ }^{1}$ AA Puchkova, ${ }^{3}$ NV Kulikova. 'Paediatrics, Kulakov Research Centre for Obstetrics, Gynecology and Perinatology; ${ }^{2}$ Pathologicoanatomy, Russian Medical University named after Pyrogov; ${ }^{3}$ Children Surgery, Filatov Children Clinical Hospital N13, Moscow, Russia

Aims To establish the role of congenital virus infection in formation of biliary atresia (BA)

Methods 75 pts age from 1 to 9 months with the following diagnoses: BA - 44, PFIC2-9, Alagille syndrome-9, bile duct cyst -7 , deficiency of a-1-antitrypsin (ZZ-phenotype)-4, perinatal sclerosing cholangitis-2, galactosemia- 1 and one patient had hepatitis as a result of congenital general acute CMV infection. Among common examinations, laboratory tests and instrumental methods, following methods were used: DNA of CMV, HSV1.2, EBV, HBV and RNA of HCV was analyzed by PCR on biopsies of the liver, blood and urine, as well as histological examination of liver biopsy performed.

Results Liver biopsy specimens were CMV DNA positive for the patient with congenital acute CMV infection, for 37 (84\%) pts with $\mathrm{BA}$, for 4 pts with Alagille syndrome, for 3 pts with bile duct cyst and for 1 child with PFIC2. EBV DNA test was positive only for 1 patient with BA and 1 with bile duct cyst. Presences of HSV1.2, HBV DNA and HCV RNA have not been found in all liver biopsy specimens. Blood samples were CMV DNA positive for the patient with congenital acute CMV infection, for $6(14 \%)$ pts with BA. Urine samples were CMV DNA positive for the patient with congenital acute CMV infection, for $7(16 \%)$ pts with BA and for 5 pts with Alagille syndrome.

Conclusion We assume that intrauterine CMV infection may play an important role in pathogenesis of BA.

\section{ARE THE 2009 ESPGHAN/ESPID'S RECOMMENDATIONS IN} ACUTE GASTROENTERITIS USED CORRECTLY?

doi:10.1136/archdischild-2012-302724.0688

${ }^{1} \mathrm{H}$ Antunes, ${ }^{2} \mathrm{D}$ Farinha, ${ }^{3} \mathrm{~V}$ Baptista. 'Gastroenterology, Hepatology and Nutrition Unit, Pediatric Department, Braga Hospital, Life and Health Sciences Research Institute 